\title{
Synthesis, Electronic Structure, and Charge Transport Characteristics of Naphthalenediimide-Based Co-Polymers with Different Oligothiophene Donor Units
}

\author{
Alessandro Luzio, Daniele Fazzi, Dario Natali, Ester Giussani, Kang-Jun Baeg, Zhihua Chen, \\ Yong-Young Noh,* Antonio Facchetti, * and Mario Caironi**
}

Received: July 8, 2013

Revised: August 27, 2013

Published online: October 29, 2013

Naphthalenediimide (NDI)-based polymers co-polymerized with thienyl units are an interesting class of polymer semiconductors because of their good electron mobilities and unique film microstructure. Despite these properties, understanding how the extension of the thienyl co-monomer affects charge transport properties remains unclear. With this goal in mind, we have synthesized a series of NDI derivatives of the parent poly $\{[N, N$-bis (2octyldodecyl)-naphthalene-1,4,5,8-bis(dicarboximide)-2,6-diyl]-alt-5,5 -(2,2 bithiophene) (P(NDI2OD-T2)), which exhibited excellent electron mobility. The strategy comprises both the extension of the donor $o$-conjugation length and the heteroatomic fusion of the thiophene rings. These newly synthesized compounds are characterized experimentally and theoretically vis-à-vis with $\mathrm{P}(\mathrm{NDI}$ 2OD-T2) as the reference. UV-vis data and cyclic-voltammetry are adopted to assess the effect of the donor modification on the frontier energy levels and on the bandgap. Intra-molecular polaronic effects are accounted for by computing the internal reorganization energy with density functional theory (DFT) calculations. Finally electrons and holes transport is experimentally investigated in field-effect transistors (FETs), by measuring currentvoltage characteristics at variable temperatures. Overall we have identified a regime where inter-molecular effects, such as the wavefunction overlap and the degree of energetic disorder, induced by the different donor group prevail over polaronic effects and are the leading factors in determining electrons mobility.

\section{Introduction}

Solution-processed organic field-effect transistors (OFETs) have been intensively investigated as they may enable new applications in low-cost, large area, printed organic electronics. ${ }^{[1-11]}$ p-type (hole-transporter) and n-type (electron-transporter) polymeric semiconductors with field effect mobilities exceeding $1 \mathrm{~cm}^{2} \mathrm{~V}^{-1} \mathrm{~s}^{-1}$ have been reported in the literature. ${ }^{[12-24]}$ However, until only very recently, the device performances of n-type polymers have been far inferior to that of p-type ones. ${ }^{[25-30]}$ In recent years, this gap has been filled thanks to the development of co-polymers based on the coupling of a weak donor unit with a strong electronaccepting one, leading to polymers with weak donor-acceptor (D-A) intra-molecular coupling and having the appropriate electronic structure for efficient electron transport. In fact, the insertion of strong electron-withdrawing units into the polymer backbone greatly enhances the electron affinity to allow stable n-type operation and efficient electron injection and transport properties.

\author{
Dr. A. Luzio, Dr. D. Fazzi, ${ }^{[+]}$Dr. D. Natali, Dr. M. Caironi \\ Center for Nano Science and Technology @PoliMi \\ Istituto Italiano di Tecnologia \\ Via Pascoli 70/3, 20133, Milano, Italy \\ E-mail: mario.caironi@iit.it \\ Dr. D. Natali \\ Dipartimento di Elettronica \\ Informazione e Bioingegneria \\ Politecnico di Milano P.za L. da Vinci, 32, 20133, Milano, Italy \\ E. Giussani \\ Dipartimento di Chimica \\ Materiali e Ingegneria Chimica CMIC "G. Natta" \\ PolitecnicodiMilano \\ P.zaLeonardodaVinci32 ，20133, Milano, Italy
}

Dr. Z. Chen, Prof. A. Facchetti

Polyera Corporation 8045 Lamon Avenue

Skokie, Illinois, 60077, USA

E-mail: afacchetti@polyera.com

Dr. K.-J. Baeg

Nano Carbon Materials Research Group

Korea Electrotechnology Research Institute (KERI)

12, Bulmosan-ro 10beon-gil, Changwon, Gyeongsangnam-do, 642-120,

Republic of Korea

Prof. Y.-Y. Noh

Department of Energy and Materials Engineering

Dongguk University

26 Pil-dong, 3-ga, Jung-gu, Seoul, 100-715, Republic of Korea

E-mail: yynoh@dongguk.edu

${ }^{[+]}$Present address: Max-Planck Institut für Kohlenforschung, Kaiser-

Wilhelm-Platz 1, D-45470, Mülheim an der Ruhr, Germany 
Imide-functionalized arenes represent one of the first and most important acceptor functionalities for n-type semiconductors, due to the high electron affinity, ${ }^{[31]}$ chemical accessibility ${ }^{[30]}$ and solubilizing ability provided by the $\mathrm{N}$-alkylation substitution. ${ }^{[32]}$ After the fi rst imide-based structures, ${ }^{[33]}$ naphthalene diimide (NDI) based co-polymers resulted in one of the most convincing approaches toward n-type semiconductors, ${ }^{[34-39]}$ thanks to the report of poly $\left\{\left[N, N^{\prime}\right.\right.$-bis(2-octyldodecyl)naphthalene-1,4,5,8-bis(dicarboximide)-2,6-diyl]-alt-5,5'-(2,2' bithiophene) (P(NDI2OD-T2)), which demonstrated large OFET electron mobilities from $\approx 0.1$ to $0.96 \mathrm{~cm}^{2} \mathrm{~V}^{-1} \mathrm{~s}^{-1}$. $[21,40,41]$ The charge transport mechanism of P(NDI2OD-T2) has been widely debated and not fully rationalized yet ${ }^{[42-46]}$ and, for this reason, $\mathrm{P}(\mathrm{NDI} 2 \mathrm{OD}-\mathrm{T} 2)$ retains attention from the scientific community, stimulating studies ranging from the molecular to the device scale.

At the molecular scale, coplanarity between the D and A units is believed to be crucial to realize an efficient integral coupling in D-A co-polymer families. ${ }^{[4]}$ Interestingly, various reports have indicated that NDI2OD (A) and T2 (D) units in $\mathrm{P}(\mathrm{NDI} 2 \mathrm{OD}-\mathrm{T} 2)$ are not coplanar, featuring a significant dihedral angle of $\approx 40^{\circ} .{ }^{48-50]}$ This interesting structural feature of $\mathrm{P}$ (NDI2OD-T2) prompted us to investigate in more detail the effect of the donor unit in NDI-based polymers on sizing both electrons and holes mobilities, clarifying the main parameters governing charge transport, and to underscore key structural parameters. Indeed the relative effect of D and A units on transport of holes and electrons is a question of general interest for the charge transport in imide-based co-polymer family. A few studies have addressed the substitution of dithiophene (T2) in P(NDI2OD-T2) with other units with the main goal of balancing ambipolarity ${ }^{[1]}$ or to understand the effect of the donor conjugation length on the electrons transport. ${ }^{[52]}$ Solubilizing side chains of the NDI group, which have a strong effect on the solid-state packing motif, have been varied as well within these studies with respect to $\mathrm{P}(\mathrm{NDI} 2 \mathrm{OD}-\mathrm{T} 2)$, partially preventing a direct comparison among the different co-polymers. In particular, several questions remain regarding the role played by the donor in defi ning: i) intra-molecular properties, such as the electron/hole reorganization energy $\left(\lambda_{\text {intra }}{ }^{\mathrm{e} / \mathrm{h}}\right),{ }^{[33-57]}$ which is a crucial parameter to account for polaronic effects; ${ }^{[58,59]}$ ii) intermolecular ones, which are strictly correlated to the films morphology and microstructure, determining the density of states for transport and electronic couplings, and which can directly be affected by backbone distortion ${ }^{[60,61]}$ as the result of $\mathrm{D}-\mathrm{A}$ dihedral angle; iii) the relative importance of these properties in sizing charge transport, thus discriminating whether intramolecular charge relaxations effects or inter-molecular ones are the most important limiting factors for carriers mobility.

With the aim of widening the understanding of this very relevant topic, in this work we investigated the effects played by the donor unit on the properties of NDI-based co-polymers by taking into account several factors, from the intra-molecular ones, to transport characteristics in field-effect devices, passing through the fi $\mathrm{lm}$ morphology. To achieve this goal, we synthesized and characterized a new series of naphthalenediimide-based derivatives of the parent polymer P(NDI2OD-T2) where the solubilizing alkyl side chains have been kept fixed (2-octyldecyl) and only the donor unit was varied based on two different strategies (Scheme 1): 1) extension of the donor conjugation by increasing the number of thiophene rings (T2 $\rightarrow$ T4); 2) heteroaromatic fusion of the thiophene rings to form thienothiophene and dithienothiophene units (T2 $\rightarrow$ TT and DTT), in order to avoid dihedral relaxations upon charging and to decrease the local contribution to the hole reorganization energy. The electrochemical redox properties and related electronic structures (HOMO/LUMO energy levels) were investigated by cyclic voltammetry, along with the solution/thin film optical absorption properties (UV-Vis). The solid state morphology of the co-polymers was investigated by atomic force microscopy (AFM) and combined IR and reflection absorption IR spectroscopy (RAIRS) techniques. Density functional theory (DFT) calculations were also employed to compute the structure and the electronic properties for each co-polymer. While our results confirm the donor efficacy in modulating the copolymer energy and optical-gaps, importantly from an intramolecular point of view, they show the significant increase in both holes and electrons reorganization energy induced by thiophene fusion. These results suggest that molecular design aiming at balancing ambipolar transport needs to carefully address charge (i.e., polaron) relaxation effects in terms of molecular structure and reorganization. Furthermore the effects of polymer structural modifications on charge carriers transport were tested in FETs devices, which revealed good electrons transporting properties as opposed to poor holes mobility. Our findings evidence that inter-molecular properties in the solid-state are strongly affected by changes in the thiophene unit and that the degree of energetic disorder induced by this modification is dominant in sizing electrons mobility over polaronic effects.

\section{Results and Discussion}

In the following sections, we first report the synthesis of the new polymers followed by a detailed characterization of their optical and electrochemical properties. Next, we investigate details of their electronic structure by DFT calculations followed by solid-state microstructural analysis carried out by atomic force microscopy and a grazing angle technique based on IRvibrational spectroscopy. ${ }^{[4]}$ Furthermore, we report on charge transport measurements carried out in field-effect transistors. Finally, we discuss the results to highlight structure-property relations and summarize the major results of this study.

\subsection{Synthesis}

NDI2OD-Br2 and P(NDI2OD-T2) were synthesized by following the previously reported procedure. ${ }^{[21]}$ The new NDIbased polymers were synthesized according to the procedures reported in Scheme 1. Briefly, all polymers, except PNDI2ODT4 were synthesized by metal-catalyzed cross coupling reaction of NDI2OD-Br2 with the corresponding $\alpha, \omega$-ditrimethylstannyl thiophene-based monomer affording the polymers in excellent yields $(>90 \%)$ after purification. Since $\alpha, \omega$-ditrimethylstannylquaterthiophene is insoluble and difficult to characterize, PNDI2OD-T4 was synthesized by first reacting NDI2OD-Br2 

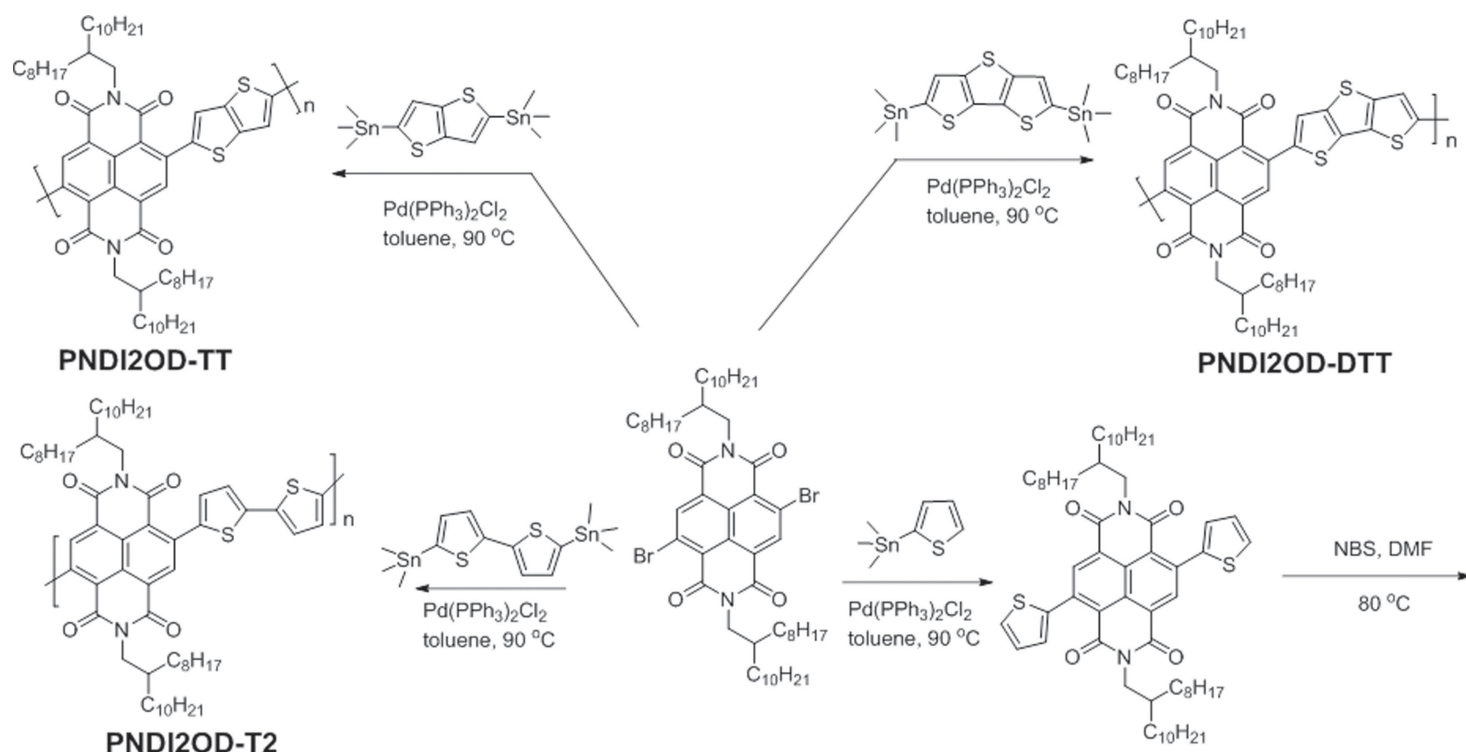

PNDI2OD-DTT

PNDI2OD-T2
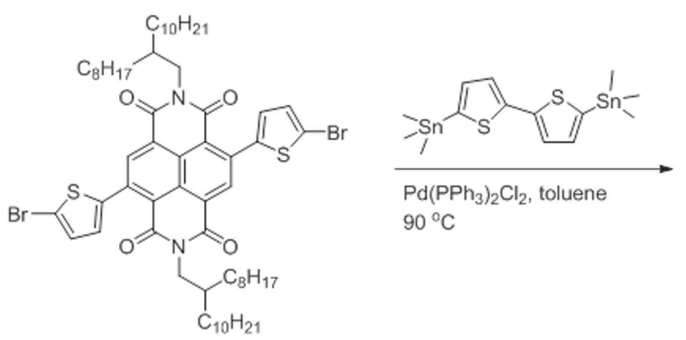

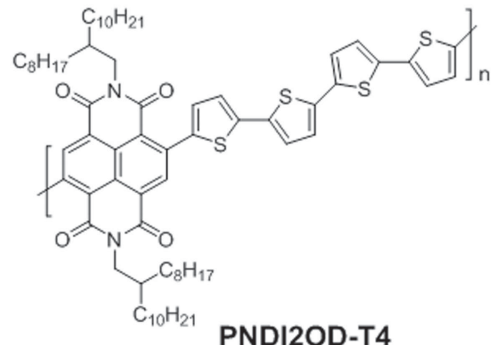

PNDI2OD-T4

Scheme 1. Synthesis of the new NDI-based polymers.

with 2-trimethylstannyl thiophene to afford NDI2OD-T2 ( $\approx 85 \%$ yields), which was then brominated under mild conditions and subsequently co-polymerized with ditrimethylstannyldithiophene to afford the polymer in excellent yields (94.1\%). All polymers were purified by multiple Soxhlet extraction followed by dissolution-precipitation and were characterized by elemental analysis, ${ }^{1} \mathrm{H}$ NM, and high-temperature GPC.

\subsection{UV-Vis and Cyclic-Voltammetry}

Figure 1a shows the absorption spectra of $\mathrm{P}(\mathrm{NDI} 2 \mathrm{OD}-\mathrm{T} 2)$, $\mathrm{P}(\mathrm{NDI} 2 \mathrm{OD}-\mathrm{TT}), \mathrm{P}(\mathrm{NDI} 2 \mathrm{OD}-\mathrm{DTT})$, and P(NDI2OD-T4) recorded in different solvents and as thin films deposited by spincoating. From a general point of view we can observe that all the spectra feature similar patterns. Each co-polymer shows two intense absorption bands attributed to the $\pi-\pi^{*}$ transition. ${ }^{[62,63]}$ The high energy band $(\approx 350-450 \mathrm{~nm})$ is assigned to excited states more localized on the thiophene units whereas the low energy broad band $(\approx 550-850 \mathrm{~nm})$ is assigned to excited states with a prevalent HOMO-LUMO contribution and classified as intra-molecular charge transfer states. The HOMO is prevalently localized on the thiophene moieties and the LUMO on the naphthalene-diimide unit. ${ }^{[48]}$

The UV-Vis absorption spectra of the NDI polymers in chloroform are broadened and red-shifted when compared to those recorded in chloronaphthalene. Furthermore, both the solution and the thin-film spectra indicate the appearance of an additional absorption peak as a shoulder of the maximum (Figure 1a). The ratio between the low and high energy peaks for the different polymers depends on the solvent employed and changes if measured on solutions or on thin films. For $\mathrm{P}(\mathrm{NDI} 2 \mathrm{OD}-\mathrm{T} 2)$, the intensity ratio of the spectral features (i.e., $\approx 800 \mathrm{~nm}, \approx 710 \mathrm{~nm}$ ) belonging to the low energy absorption has been recently ascribed to solution/solid-state polymer aggregation. ${ }^{[64]}$ Thus, a similar phenomenon may account for the spectral variations of the new NDI-based polymers; however, detailed spectroscopic studies are ongoing to investigate in detail this point, which is currently out of the scope of this investigation. If we compare spectra in chloronaphthalene, which are less affected by aggregation, we observe that by changing the donor units, the UV-Vis spectra show variations which can be ascribed, at least partially, to changes in polymer backbone $\pi$-conjugation. Thus P(NDI2OD-TT) exhibits two absorption bands located at higher energies than those of the other polymers since the TT unit has the lowest number of $\pi$-electrons within the donor series. P(NDI2OD-DTT) has an absorption spectrum similar to the parent P(NDI2OD-T2) polymer, and it shows also the same number of $\pi$-electrons on the thiophene units. P(NDI2OD-T4) is the co-polymer featuring the donor unit with the largest number of $\pi$-electrons and, indeed, the two absorption bands are at lower energies with 

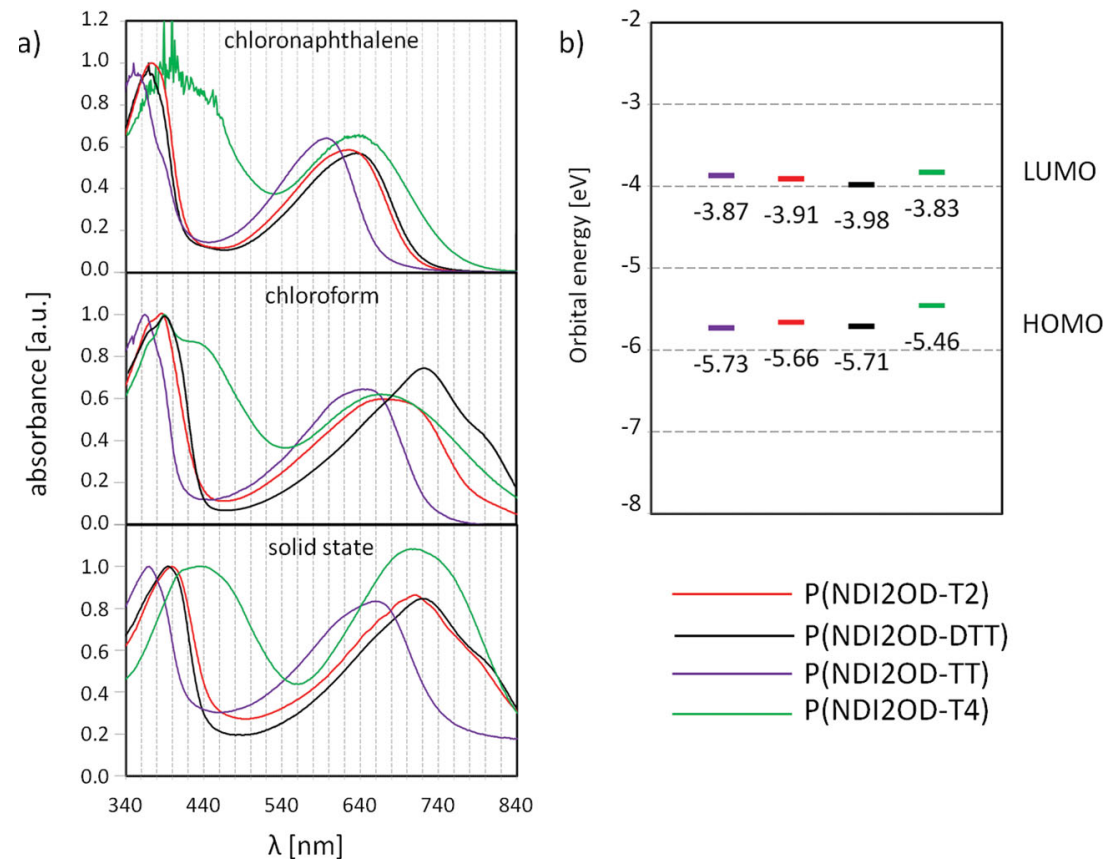

Figure 1. a) UV-Vis spectra of $P(N D I 2 O D-T 2), P(N D \mid 2 O D-T T), P(N D I 2 O D-D T T)$, and $\mathrm{P}(\mathrm{NDI}$ 2OD-T4) in chloronaphthalene and chloroform or as thin films. b) Plot of the HOMO and LUMO energy estimated by cyclic voltammetry (LUMO) and UV-Vis investigation (HOMO).

respect to the other polymers. In particular, for P(NDI2OD-T4) the high energy absorption band (i.e., excited states more localized upon T4 units) is red shifted with respect to that of the other compounds due to the enhanced $\pi$-electron conjugation.

Cyclic voltammetry scans, which are reported in the Supporting Information, exhibit two reversible reductions for all the compounds, as observed for P(NDI2OD-T2). ${ }^{[63]}$ By combining the electrochemical with the optical data recorded in chloronaphthalene solution the HOMO/LUMO energies were estimated following the method described in Supporting Information. These values are summarized in Figure $1 \mathrm{~b}$. Interestingly, a very limited dependence of the LUMO levels on the thiophene co-monomer structure is found, consistently with the LUMO preferential localization on the NDI2OD unit. ${ }^{[62,63]}$ As expected, the band gap $\left(E_{\mathrm{G}}\right)$ follows the trend imposed by the different $\pi$-conjugation extension of the compounds.

\subsection{DFT Structures and Intra-Molecular Reorganization Energies}

In this section structural and electronic properties of the newly designed co-polymers have been investigated through DFT calculations. The equilibrium structures of the neutral as well as the negatively and positively charged species are calculated to unveil the effect of the different donor groups. The aim is to quantify the polaronic effects in these polymers, by computing the spatial localization of the charged species and their energetic cost, described by the internal reorganization energy $(\lambda)$.

Intra-molecular equilibrium structures have been optimized considering, for each compound, the neutral and charged (both anion and cation) species at the (U) CAM-B3LYP/6-31G* level through an oligomer approach, spanning from $n=1$ to $n=7(\mathrm{D}-\mathrm{A})_{n}$ units. The structural parameters we considered for the analysis are the dihedral angle $\theta$ between the $\mathrm{D}$ and $\mathrm{A}$ units, and in the case of P(NDI2OD-T2) and P(NDI2OD-T4), the torsional angle $\tau$ between the thiophene rings. Details about the optimized geometries in each electronic state are reported in the Supporting Information, along with the bond lengths relaxations $(\Delta R)$ upon charging for the longest oligomer (i.e., $n=7$ ), showing the polaron structural confinement. An important point emerging from the equilibrium geometries optimized in the neutral state is that, as in the case of other studies, ${ }^{[48-50]}$ in all co-polymers the NDI and the thiophene-based units are not coplanar but show a significant $\theta$, ranging from $38^{\circ}$ to $43^{\circ}$.

The computed structural relaxations upon electron/hole charging are fundamental for rationalizing the reorganization energy values reported in Figure 2. As discussed elsewhere, ${ }^{[48]}$ the torsional relaxation $(\Delta \tau)$ upon positive charging and the lower $\pi$-electron conjugation of the T2 unit with respect to the NDI2OD, contribute to an overall raise of $\lambda_{\text {intra }}^{\mathrm{h}}$ with respect to the electron one for P(NDI2OD-T2), featuring $\lambda_{\text {intra }}^{e} \approx 0.32 \mathrm{eV}$, that is almost two times lower than the hole one $\left(\lambda_{\text {intra }}^{\mathrm{h}} \approx 0.52 \mathrm{eV}\right)$. From a
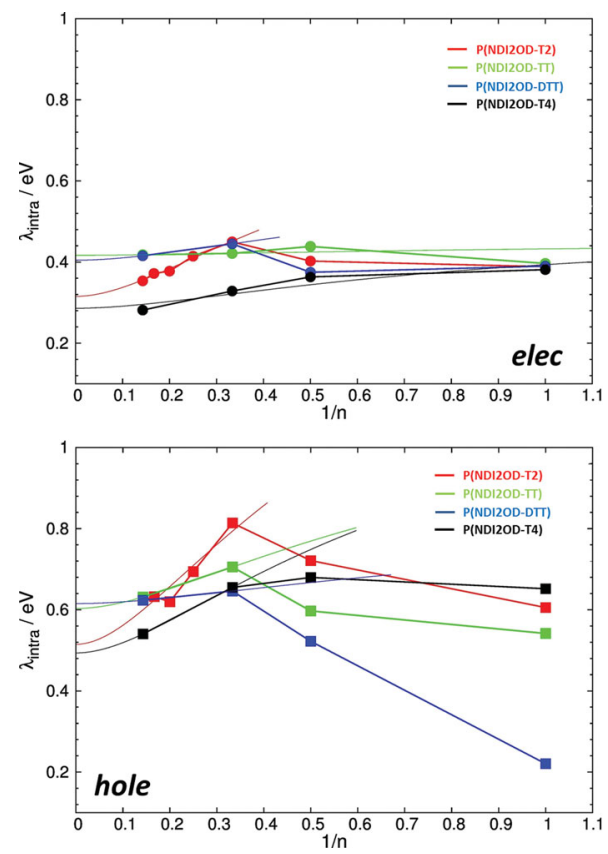

Figure 2. (U)CAM-B3LYP/6-31G* computed intra-molecular electron (top panel) and hole (bottom panel) reorganization energies (symbols) and asymptotic fits to $n=\infty$ (lines) for P(NDI2OD-T2) (red, data from Ref. [48]), P(NDI2OD-TT) (green), P(NDI2OD-DTT) (blue) and P(NDI2ODT4) (black). Circles refer to $\lambda_{\text {intra }}^{\text {e }}$ while squares refer to $\lambda_{\text {intra }}^{\text {h }}$. 
Table 1. Extrapolated polymer limit $(n \rightarrow \infty)$ values for the reorganization energies.

\begin{tabular}{lcc}
\hline & $\lambda_{\text {intra }}^{\mathrm{e}}[\mathrm{eV}]$ & $\lambda_{\text {intra }}^{\mathrm{h}}[\mathrm{eV}]$ \\
\hline $\mathrm{P}$ (NDI2OD-T2) & 0.32 & 0.52 \\
$\mathrm{P}($ NDI2OD-TT) & 0.40 & 0.60 \\
$\mathrm{P}($ NDI2OD-DTT) & 0.40 & 0.60 \\
$\mathrm{P}$ (NDI2OD-T4) & 0.28 & 0.52 \\
\hline
\end{tabular}

qualitative point of view, by looking at the donor monomers in the newly synthesized co-polymers, we would therefore expect a

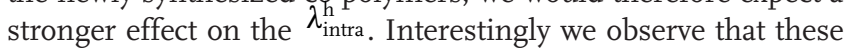
simple expectations are fulfilled only on the monomers $(n=1)$ species, while for oligomers approaching the polymer limit $(n>4)$, the modification of the donor unit has a clear effect on $\lambda_{\text {intra }}^{e}$ as well, which is not trivial to predict without recurring to ab-initio calculations.

For $\mathrm{P}(\mathrm{NDI} 2 \mathrm{OD}-\mathrm{T} 2), \mathrm{P}(\mathrm{NDI} 2 \mathrm{OD}-\mathrm{TT})$, and $\mathrm{P}(\mathrm{NDI} 2 \mathrm{OD}-\mathrm{DTT})$ similar trends of $\lambda_{\text {intra }}^{\mathrm{e} / \mathrm{h}}$ versus $1 / n$ were computed, featuring a $\lambda_{\text {intra }}^{\mathrm{e} / \mathrm{h}}$ maximum at $n=3$, corresponding to the polaron-length structural reorganization (see Supporting Information). For $\mathrm{P}(\mathrm{NDI} 2 \mathrm{OD}-\mathrm{T} 4)$ hole/electron reorganization energies trends do not show the same maximum at $n=3$, but they show a plateau around $n=2-3$, while decreasing for longer oligomers. The extrapolated polymer limit $(n \rightarrow \infty)$ values for the reorganization energies are summarized in Table 1. Co-polymers featuring thiophene condensed rings (T'T and DTT) have extrapolated hole/electron reorganization energies higher than $\mathrm{P}(\mathrm{NDI} 2 \mathrm{OD}-\mathrm{T} 2)$, while $\mathrm{P}(\mathrm{NDI} 2 \mathrm{OD}-\mathrm{T} 4)$ exhibits reorganization energies quite similar to P(NDI2OD-T2), with both $\lambda_{\text {intra }}^{\text {h }}$ and $\lambda_{\text {intra }}^{e}$ slightly lower than those of P(NDI2OD-T2). Note that the computed reorganization energies appear to challenge simple arguments regarding the effect of the modification of the donor unit: for example, it could be reasonable to assume that by condensing the thiophene rings (TT and DTT cases) one could reduce the hole reorganization energy as an effect of annellation (i.e., avoiding $\Delta \tau$ relaxations), while slightly affecting the electron reorganization term. By analyzing each oligomer we observe that, while $\lambda_{\text {intra }}^{e}$ for each monomeric species (i.e., $n=1)$ has similar values $(\approx 0.40 \mathrm{eV})$ because electrons relax mainly on NDI2OD unit, $\lambda_{\text {intra }}^{\text {h }}$ are different. In particular for $n=1, \mathrm{P}$ (NDI2OD-T2) shows a hole reorganization energy of $0.60 \mathrm{eV}$ (equal to P(NDI2OD-T4) species), while P(NDI2ODTT) and $\mathrm{P}(\mathrm{NDI} 2 \mathrm{OD}-\mathrm{DTT})$ show $0.53 \mathrm{eV}$ and $0.20 \mathrm{eV}$ respectively. The monomer of $\mathrm{P}(\mathrm{NDI} 2 \mathrm{OD}-\mathrm{DT} T)$ has a hole reorganization energy markedly lower than the others, thus supporting the idea of reducing $\lambda_{\text {intra }}^{\mathrm{h}}$ by suppressing any thiophene internal torsional relaxations upon hole charging through ring condensation. Since our materials are low-band gap co-polymers, the monomer unit cannot be considered as a good model for predicting the polymer electronic properties, hence longer oligomers have to be considered. In this regards, as previously discussed, $\lambda_{\text {intra }}^{\mathrm{h}}$ for thiophene-condensed species increases till the peculiar defect size (i.e., polaron size) oligomer length ( $n$ $=3-4)^{[48,65]}$ and then it decreases according to the extended $\pi$ electron conjugation up to the asymptotic values that result to be higher than that of P(NDI2OD-T2).
From the intra-molecular reorganization energy results we can conclude that each NDI-thiophene based co-polymers show electron reorganization energies lower than the hole ones, and that fusion of thiophene rings, to avoid any thiophene torsional relaxation upon charging, does not decrease the hole reorganization energy because $\pi$-electron conjugation of the thiophene units remains quite low. DFT computations also show that increasing the number of thiophene rings from P(NDI2OD-T2) to $\mathrm{P}(\mathrm{NDI} 2 \mathrm{OD}-\mathrm{T} 4)$ does not result in a significant lowering of the hole reorganization energies and only slightly affects the electron one. Overall P(NDI2OD-TT) and P(NDI2OD-DTT) show both hole and electron reorganization energies $0.10 \mathrm{eV}$ higher than P(NDI2OD-T2) and P(NDI2OD-T4).

From this first DFT screening, P(NDI2OD-T2) and $\mathrm{P}(\mathrm{NDI} 2 \mathrm{OD}-\mathrm{T} 4)$ are the polymers showing the lowest reorganization energies. In a scenario of low energetic disorder, where the reorganization energy could play a major role in determining the field-effect mobility, these two co-polymers are expected to show the best FET performances. Obviously, the capability of these polymers to assemble in the solid state, producing more or less strong coupling integrals, and the particular density of states, with its characteristic energetic disorder, will play a crucial role as well in determining the transport properties. Importantly, in the case of a strong energetic disorder, polaronic effects are expected to play a minor role. ${ }^{66]}$ To elucidate this point, structural characterizations of the thin films are investigated in the next section and, in order to unveil whether polaronic effects or disorder prevail in determining mobility values (Section 2.5 ), variable temperature $I-V$ characteristics are analyzed in Section 2.6.

\subsection{Films Morphology Characterization Through AFM and IR Spectroscopy}

In this section, NDI co-polymer thin films, which are then used to fabricate OFETs (vide infra) are characterized by AFM and IR spectroscopy to study how these compounds pack in the solid state. DFT computations clearly suggested the presence of an intra-molecular dihedral angle of $\approx 38^{\circ}$ between the NDI and thiophene units in all the co-polymers (vide supra). Thus, a significant torsional angle is expected for these polymers also in the solid-state, given the large energetic barrier for the complete planarization of the donor and acceptor units. ${ }^{[49]}$ Significant differences in this angle for the investigated co-polymers should correspond to different packing motifs in the films.

The AFM images of the NDI-based polymer thin films deposited by spin coating from dichlorobenzene on glass (Figure 3) clearly show different surface morphologies. Stacking of rigid elongated domains, hundreds of $\mathrm{nm}$ long and 3-4 $\mathrm{nm}$ large, were observed for the very flat P(NDI2OD-T2) film surface. A root mean square roughness $\left(R_{\mathrm{RMS}}\right)$ of $0.65 \mathrm{~nm}$ was calculated on a scan size of $1 \mu \mathrm{m} \times 1 \mu \mathrm{m}$. The surface of P(NDI2ODTT) show similar features as those of P(NDI2OD-T2) films, although they are rather less defined; moreover a higher surface roughness was calculated $\left(R_{\mathrm{RMS}}=0.81 \mathrm{~nm}\right.$, scan size $1 \mu \mathrm{m} \times 1 \mu \mathrm{m}) . \mathrm{P}(\mathrm{NDI} 2 \mathrm{OD}-\mathrm{DTT})$ surface $\left(R_{\mathrm{RMS}}=1.62 \mathrm{~nm}\right.$, scan size $1 \mu \mathrm{m} \times 1 \mu \mathrm{m})$ exhibits a pattern characterized by small and nodular features whereas P(NDI2OD-T4) films show a rough 
a)

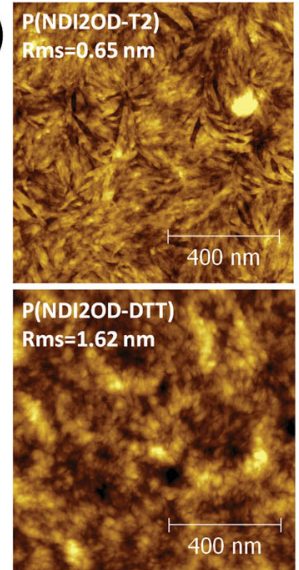

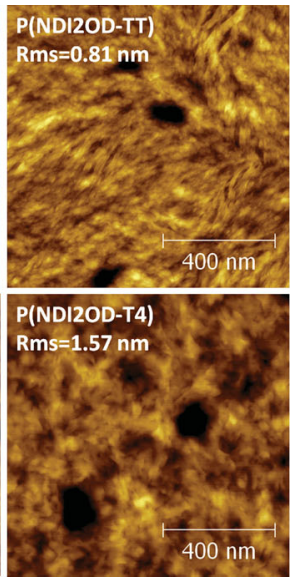
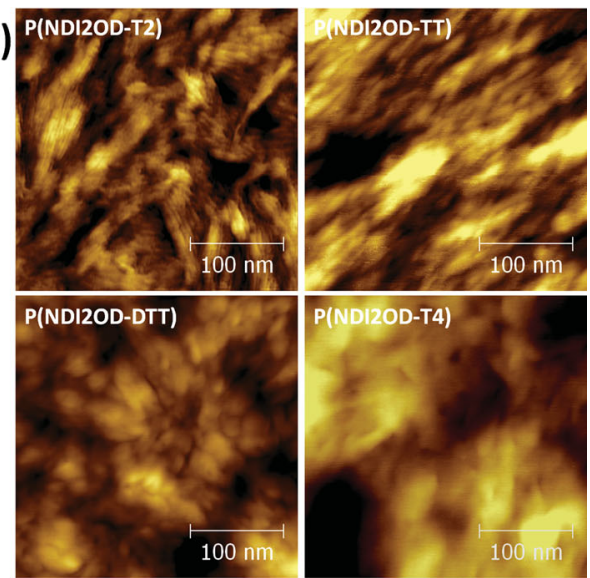

Figure 3. AFM Topography of $\mathrm{P}(\mathrm{NDI}$ 2OD-T2), $\mathrm{P}(\mathrm{NDI}$ 2OD-TT), $\mathrm{P}(\mathrm{NDI}$ 2OD-DTT) and $\mathrm{P}(\mathrm{NDI}$ 2OD-T4) thin films. a) scan sizes of $1 \mu \mathrm{m} \times 1 \mu \mathrm{m}$ and b) of $300 \mathrm{~nm} \times 300 \mathrm{~nm}$.

and almost featureless surface $\left(R_{\mathrm{RMS}}=1.57 \mathrm{~nm}\right)$. From the AFM data it results that $\mathrm{P}(\mathrm{NDI} 2 \mathrm{OD}-\mathrm{T} 2)$ and $\mathrm{P}(\mathrm{NDI} 2 \mathrm{OD}-\mathrm{TT})$ are, amongst all compounds, the ones showing the flattest surface, organized in elongated and packed domains.

To gain further insights into the structural and molecular features of these polymers, a technique which combines transmission IR spectroscopy and RAIRS was utilized to detect the polymer local orientation. ${ }^{[49]}$ Figure 4 reports the comparisons between the transmission IR spectra (i.e., radiation polarized in the substrate plane, that is, $(x, \gamma)$ plane) and the RAIRS (i.e., radiation polarized perpendicular to the substrate, that is, $z$ direction) for all co-polymers. For P(NDI2OD-T2) we recently reported that four main IR active bands can be utilized for defining the intra-molecular structure and orientations of the polymer backbone. ${ }^{[49]}$ Namely: a and b bands, corresponding to the $\mathrm{C}=\mathrm{O}$ symmetric and antisymmetric stretching, $\mathbf{c}$ and $\mathbf{d}$ bands corresponding to the out of plane (OPLA) CH mode of the T2 unit and the CS stretching respectively. These bands feature
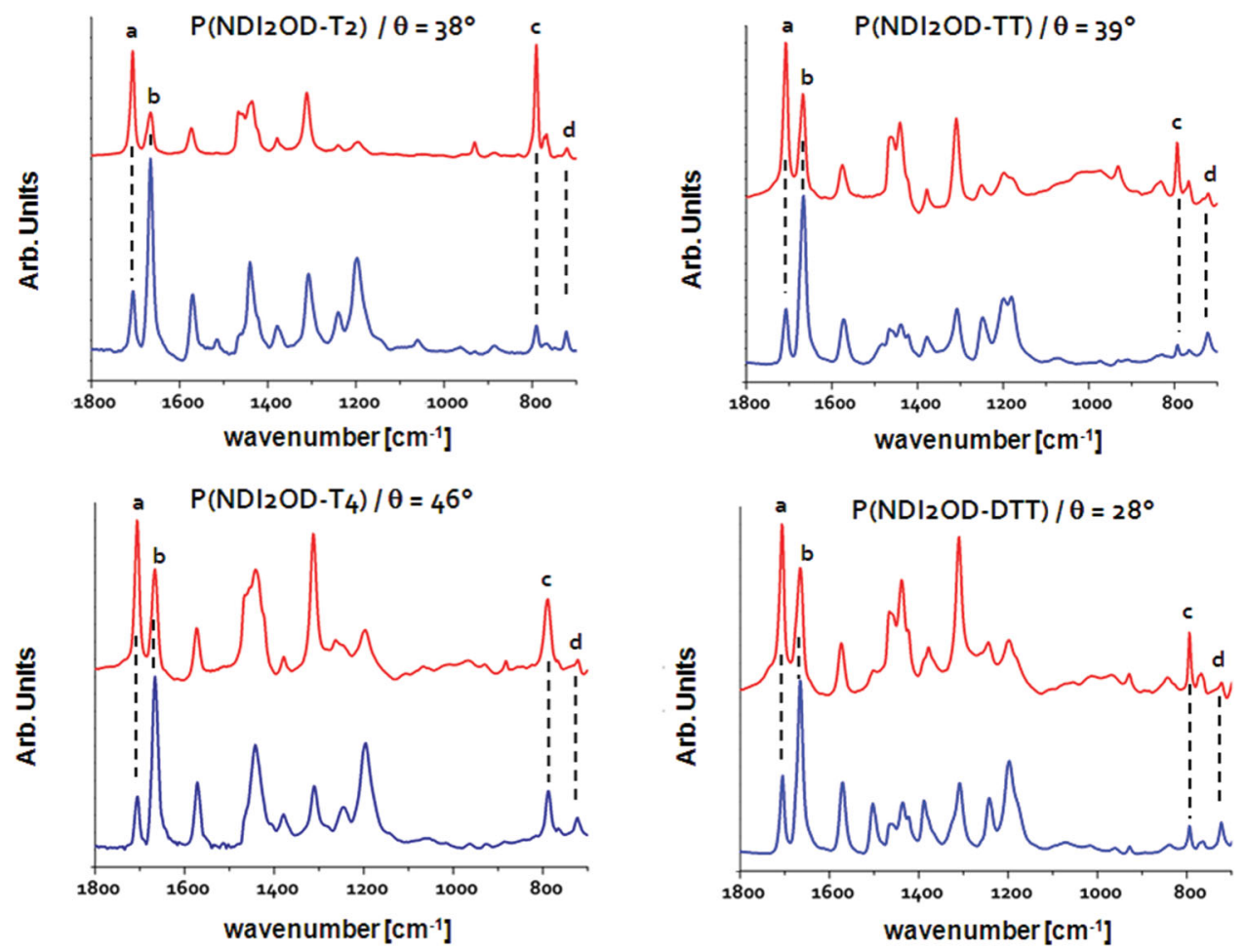

Figure 4. Transmission IR (blue line) and RAIRS (red line) spectra recorded on P(NDI2OD-T2), P(NDI2OD-TT), P(NDI2OD-DTT), and P(NDI2OD-T4) thin films deposited on Au. As insets, the main IR active bands (a-d) and the dihedral angle between NDI2OD and the thiophene-based unit evaluated from the analysis of the IR/RAIRS intensities are reported. 
different polarizations: $\mathbf{a}$ is polarized along the NDI2OD long axis, $\mathbf{b}$ along the NDI2OD short axis, that is, the polymer chain, $\mathbf{c}$ is perpendicular to the T2 local plane and $\mathbf{d}$ is in the T2 plane. Based on these assignments and considering the RAIRS spectrum (Figure 4), which shows an enhancement with respect to transmission IR of the $\mathbf{c}$ and $\mathbf{a}$ intensities and a decrease of the b and $\mathbf{d}$ intensities, we have confi rmed here that P(NDI2ODT2) backbone lies preferentially parallel to the substrate surface, featuring T2 units $\mathrm{fl}$ at to the surface while NDI2ODs are tilted with an angle of $38^{\circ}$. Following the same approach, we have analyzed IR and RAIRS spectra of the new polymers, unveiling similar features: i) all compounds show similar IR spectra in the region of $\mathrm{C}=\mathrm{O}$ stretchings ( $\mathbf{a}$ and $\mathbf{b}$ bands) and OPLA normal modes (c band); ii) RAIRS spectra present an enhancement of the $\mathbf{a}$ and $\mathbf{c}$ bands featuring a main polarization perpendicular to the substrate; iii) the three compounds present a more intense $\mathbf{b}$ band in RAIRS spectra, meaning that a fraction of the polymer chains does not lay mainly (and on average over the whole fi $\mathrm{lm}$ thickness) parallel to the surface thus showing a component out of the plane. Among them, P(NDI2OD-TT) shows however distinctive features that suggest an alignment of the backbone very close to the P(NDI2OD-T2) case, while $\mathrm{P}(\mathrm{NDI} 2 \mathrm{OD}-\mathrm{DTT})$ and $\mathrm{P}(\mathrm{NDI} 2 \mathrm{OD}-\mathrm{T} 4)$ strongly deviate from this picture.

$\theta$ has been experimentally derived also for the other copolymers. As reported in Figure 4, while P(NDI2OD-T2) and $\mathrm{P}(\mathrm{NDI} 2 \mathrm{OD}-\mathrm{TT})$ show similar $\theta$ values $\left(38^{\circ}\right.$ and $\left.39^{\circ}\right), \mathrm{P}(\mathrm{NDI} 2 \mathrm{OD}-$ DTT) and $\mathrm{P}(\mathrm{NDI} 2 \mathrm{OD}-\mathrm{T} 4)$ present markedly different values, $28^{\circ}$ and $46^{\circ}$ respectively. As mentioned above, a different dihedral angle $\theta$ is indicative of a different packing capability of the chains in the solid state, and this can have a strong influence on charge transport characteristics. Although the reported investigations do not allow us to access the detailed solid state packing of the polymers in the thin films under study, we can observe that the co-polymers showing a similar dihedral angle around $40^{\circ}$ and a backbone mainly parallel to the substrate, which is the case of $\mathrm{P}(\mathrm{NDI} 2 \mathrm{OD}-\mathrm{T} 2)$ and $\mathrm{P}(\mathrm{NDI} 2 \mathrm{OD}-\mathrm{TT})$, are characterized by similar AFM surface features and by a very limited surface roughness. Deviations of $\theta$ from this value and of the preferential direction of the backbone lead to evidently different AFM features and to a rougher surface as in the case of P(NDI2OD-DTT) and P(NDI2OD-T4) (Figure 3).

\subsection{Room-Temperature $I-V$ Characteristics of Field-Effect Transistors}

All NDI co-polymers under study were tested in FET devices to measure their electrons $\left(\mu_{\mathrm{e}}\right)$ and holes $\left(\mu_{\mathrm{h}}\right)$ field-effect mobility. Figure 5a shows the our FET structure consisting of a staggered top-gate, bottom-contact (TGBC) geometry with photolithographically defined gold Source and Drain electrodes on a glass substrate. Two different polymeric dielectric layers were employed in this work: PMMA $(k=3.6)$ and Cytop $(k=2.1)$. All the semiconductors were deposited by spin coating of dichlorobenzene solutions. All the curves reported in Figure 5b-i were measured on PMMA based devices, in which the semiconductor underwent a thermal treatment of $200{ }^{\circ} \mathrm{C}$ prior to the dielectric deposition. We also studied semiconductor thin films which were subjected to $120^{\circ} \mathrm{C}, 150{ }^{\circ} \mathrm{C}$ thermal treatments: no difference was observed in the electron transport behavior, however, some influence could be noticed for the holes transport only (see also Supporting Information). Figure 6b-e shows almost ideal n-type transfer curves for the
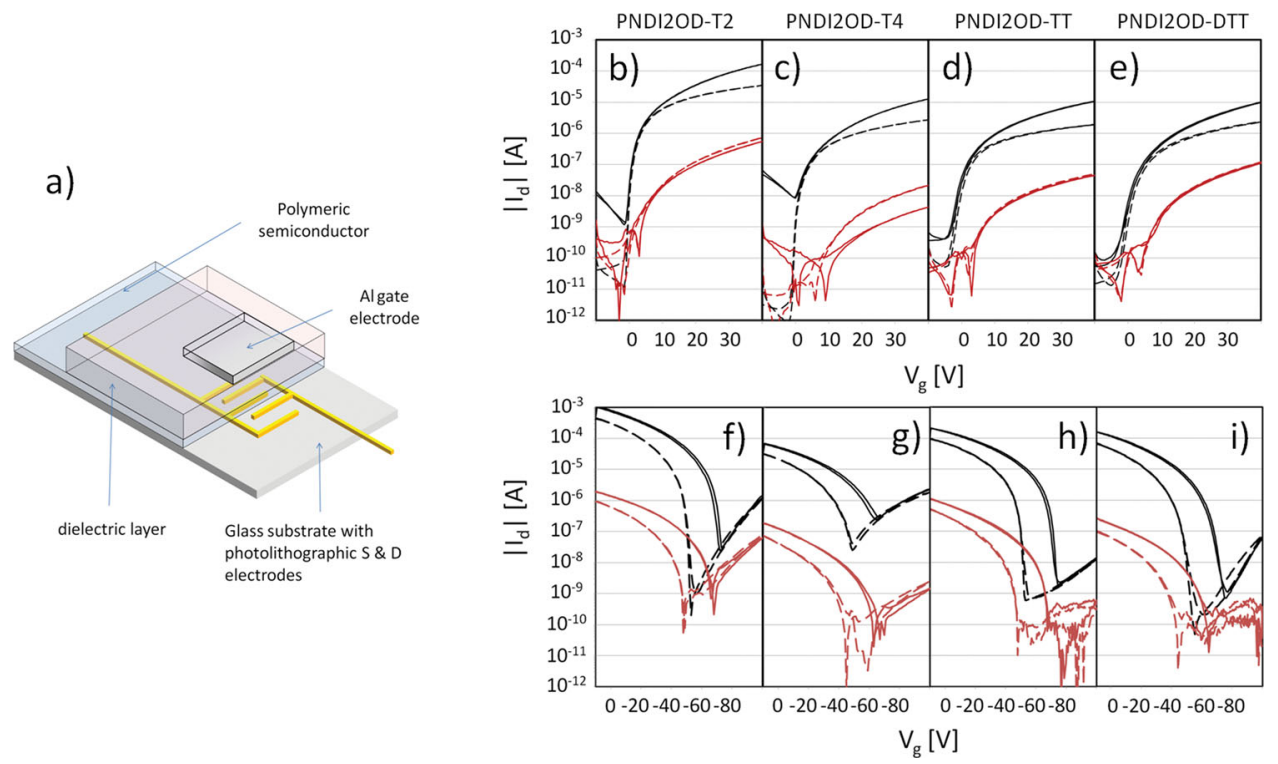

Figure 5. a) Schematic representation of the staggered OFETs realized and characterized in this work. $\mathrm{N}$-channel transfer characteristics of devices based on b) P(NDI2OD-T2), c) P(NDI2OD-T4), d) P(NDI2OD-TT), and e) P(NDI2OD-DTT) with PMMA as the dielectric layer; dashed and continuous lines refer to the linear $\left(V_{d}=5 \mathrm{~V}\right)$ and the saturation regime $\left(V_{d}=40 \mathrm{~V}\right)$, respectively. $P$-channel transfer characteristics of devices based on f) P(NDI2OD-T2), g) P(NDI2OD-T4), h) P(NDI2OD-TT), and i) P(NDI2OD-DTT) with PMMA as the dielectric layer; $V_{d}=-50 \mathrm{~V}$ (continuous lines) and $V_{d}=-70 \mathrm{~V}$ (dashed lines) were applied during the measurement. In all the plots the red lines represent the gate leakage currents. 
three devices measured at room temperature (RT), with relatively low subthreshold swing $\left(1 \div 2 \mathrm{~V} \mathrm{dec}^{-1}\right)$ given the low gate capacitance $\left(\approx 5 \mathrm{nF} \mathrm{cm}{ }^{-2}\right)$ of the test device adopted, and approximately zero turn-on voltage. This is certainly a clue of the good quality of the semiconductor-dielectric interface in all cases from an electron transport point of view. Moreover, a good electron injection (extraction) from gold source (drain) is observed, since the output curves (see Supporting Information) exhibit almost linear trend in the proximity of $V_{\mathrm{d}}=0 \mathrm{~V}$. By comparing the drain currents of Figure 5b-e at $V_{\mathrm{d}}=V_{\mathrm{g}}=$ $40 \mathrm{~V}$ it is already possible to observe that, among the newly synthesized polymers, the devices based on P(NDI2OD-DTT) exhibit the highest $\mu_{\mathrm{e}}$ when a PMMA dielectric is adopted $\left(\mu_{\mathrm{e}}=0.04 \pm 0.01 \mathrm{~cm}^{2} \mathrm{~V}^{-1} \mathrm{~s}^{-1}\right)$, followed by the OFET based on $\mathrm{P}$ (NDI2OD-TT) $\left(\mu_{\mathrm{e}}=0.02 \pm 0.01 \mathrm{~cm}^{2} \mathrm{~V}^{-1} \mathrm{~s}^{-1}\right)$. Devices based on $\mathrm{P}(\mathrm{NDI} 2 \mathrm{OD}-\mathrm{T} 4)$ show the lowest electrons mobility $\left(\mu_{\mathrm{e}}=\right.$ $\left.0.01 \pm 0.002 \mathrm{~cm}^{2} \mathrm{~V}^{-1} \mathrm{~s}^{-1}\right)$. When Cytop is employed as the dielectric layer, $\mathrm{P}(\mathrm{NDI} 2 \mathrm{OD}-\mathrm{TT})$ saturation mobility increases to $0.04 \pm 0.01 \mathrm{~cm}^{2} \mathrm{~V}^{-1} \mathrm{~s}^{-1}$ and P(NDI2OD-DTT) saturation mobility decreases to $0.02 \pm 0.004 \mathrm{~cm}^{2} \mathrm{~V}^{-1} \mathrm{~s}^{-1}$. Only the saturation mobility of $\mathrm{P}(\mathrm{NDI} 2 \mathrm{OD}-\mathrm{T} 4)$ is not substantially affected upon changing the dielectric layer, still resulting the lowest among the three systems (Figure6). In accordance with the output curves described above, from an analysis of the contact resistances (details will be reported elsewhere) it was found that the apparent mobility values here reported are generally very close to the intrinsic mobility values, with a difference within 20-30\% range, as an effect of a relatively small contribution of contact resistances for all the systems investigated. The different mobility trends evidence the complexity of the infl uence of the dielectric-semiconductor interface on the device performances. In fact, despite Cytop low dielectric constant should generally enable a more energetically ordered interface with respect to PMMA, leading to a more ideal accumulation and transport mechanism, ${ }^{[67]}$ a different intermixing of the semiconductor and dielectric interface, due to solvents driven interactions during the dielectric layer deposition, can also strongly infl uence the available density of states. ${ }^{[42]}$ For example in the case of P(NDI2OD-T2), a thicker interfacial width (i.e., a rougher interface), generally considered a detrimental factor for effi cient OFET transport, was demonstrated in the case of Cytop. ${ }^{[68]}$ The concurrence of these two distinct factors can partially hinder the effect of the different insulator dielectric constant and might partially explain the different trends observed with the two dielectrics.
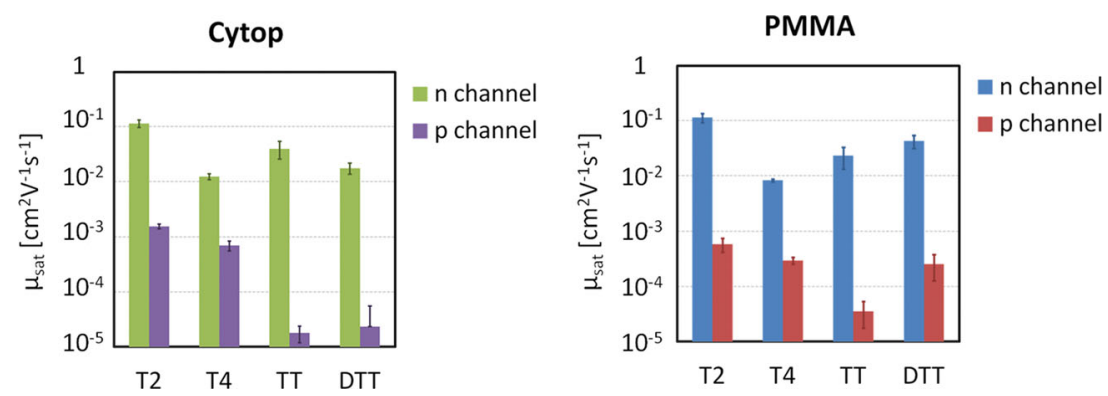

Figure 6. Histograms summarizing the $n$-channel and $p$-channel field effect mobility in the saturation regime of OFETs realized in this work.
However, our results so far indicate that, irrespectively of the adopted dielectric, P(NDI2OD-TT) and P(NDI2OD-DTT) devices show similar electrons mobility, always higher than the P(NDI2OD-T4) case (Figure 6). All derivatives show lower electrons mobility compared to that of P(NDI2OD-T2), which is $\approx 0.1 \mathrm{~cm}^{2} \mathrm{~V}^{-1} \mathrm{~s}^{-1}$. This indicates that structural changes in the thiophene-based D units are unfavorable for electron transport.

$P$-channel characteristics were also investigated (Figure $5 \mathrm{f}-\mathrm{i}$ ). For all devices the holes mobilities are much lower than the electron mobilities. With a PMMA dielectric, the extracted saturation $\mu_{\mathrm{p}}$ are $3.5 \times 10^{-5} \pm 1.8 \times 10^{-5} \mathrm{~cm}^{2} \mathrm{~V}^{-1} \mathrm{~s}^{-1}, 2.5 \times 10^{-4} \pm 1.3 \times$ $10^{-4} \mathrm{~cm}^{2} \mathrm{~V}^{-1} \mathrm{~s}^{-1}$ and $2.95 \times 10^{-4} \pm 4.1 \times 10^{-5} \mathrm{~cm}^{2} \mathrm{~V}^{-1} \mathrm{~s}^{-1}$ for $\mathrm{P}$ (NDI2OD-TT), P(NDI2OD-DTT), and P(NDI2OD-T4), respectively (Figure 6 ). Again, these $\mu_{\mathrm{p}}$ values are generally inferior to that of P(NDI2OD-T2) $\left(6.5 \times 10^{-4} \pm 1.3 \times 10^{-4} \mathrm{~cm}^{2} \mathrm{~V}^{-1} \mathrm{~s}^{-1}\right)$. It must be mentioned that $p$-channel behavior of devices based on P(NDI2OD-TT) and P(NDI2OD-DTT) are influenced by the semiconductor film thermal annealing: holes mobilities lower than $10^{-6} \mathrm{~cm}^{2} \mathrm{~V}^{-1} \mathrm{~s}^{-1}$ were measured for devices annealed at $T<200{ }^{\circ} \mathrm{C}$ (see Supporting Information). This result suggests that a morphological rearrangement induced by the thermal treatment might improve hole transport in these semiconductors. On the other hand, hole transport for devices based on $\mathrm{P}(\mathrm{NDI} 2 \mathrm{OD}-\mathrm{T} 2)$ and $\mathrm{P}(\mathrm{NDI} 2 \mathrm{OD}-\mathrm{T} 4)$ is insensitive to thermal treatments. The use of Cytop substantially affects only the mobility of P(NDI2OD-DTT) devices, decreasing the holes mobility by an order of magnitude. In general, these findings evidence a strongly unbalanced ambipolarity, where the holes mobility is $\approx 100-1000 \times$ lower than that of electrons. Interestingly, it is the P(NDI2OD-T4) device which shows the highest holes mobility, with a value closer to the one obtained for P(NDI2OD-T2) devices. We note that our DFT calculations would predict an impeded hole transport with respect to electrons, in agreement with the asymmetric transport found in bulk diode devices for P(NDI2OD-T2), ${ }^{[50]}$ and that P(NDI2ODT2) and P(NDI2OD-T4) lower reorganization energies would favor hole mobility with respect to the other NDI co-polymers. However this result should be considered with care since in the case of field-effect devices based on NDI polymers hole injection is very limited, as previously evidenced for P(NDI2ODT2). ${ }^{[50,69]}$ This limitation, along with the high threshold voltages $(<-50 \mathrm{~V}$ for all the series) and consequently the low on-currents, preclude accurate estimation of the intrinsic mobility values. Qualitatively, having a lower HOMO level (Figure 1b), $\mathrm{P}(\mathrm{NDI}$ 2OD-T4) could actually benefit from a lower hole injection barrier with respect to the case of the other derivatives. Further in depth analysis would be required to disentangle the various effects, but unfortunately a reliable extraction of contact resistances for holes could not be achieved, due to the very low on-current levels, which also make low temperature measurements unviable. For this reason in the following sections we focus on electrons transport.

In summary, the FETs based on the newly synthesized co-polymers show to have good electrons transporting properties, while 
having very limited holes transport properties, at least in the tested device configurations. While from the previous structural analysis it is not surprising that P(NDI2ODTT) devices show a closer electron mobility to $\mathrm{P}(\mathrm{NDI} 2 \mathrm{OD}-\mathrm{T} 2)$, given the similar surface morphology and the similar dihedral angle between the NDI and T2 unit, it is less obvious why P(NDI2OD-DTT) should achieve similar transport properties. In the case of P(NDI2OD-T4), despite intra-molecular properties, that predict lower polaronic effects, poorer performances are obtained, probably refl ecting an impeded transport due to morphological issues, as suggested by

AFM and IR analysis. In any case other relevant factors are to be taken into account and more insight in the device physics is required. In the next section, we therefore investigate the polymers density of states and the degree of interchain coupling by variable temperature measurements on FETs.

\subsection{Variable-Temperature $I-V$ Measurements}

To obtain a more quantitative description of NDI polymer electrons transport properties, we performed variable temperature measurements. To this end, the intrinsic mobility in the saturation regime was extracted and to take into account the effect of contact resistance, the differential method (DM) was utilized. ${ }^{[70]}$ Within the application of DM, we have modeled the field-effect mobility as a power law of $V_{\mathrm{g}}$, viz. $\mu=\mu_{0}\left(V_{\mathrm{g}}-V_{\mathrm{T}}\right)^{\gamma}$. We found a marked dependence on $V_{\mathrm{g}}$, with $\gamma$ being in excess of 1 for $T<250 \mathrm{~K}$, thus implying a large dependence of the mobility on the charge carrier density. This cannot be explained within a polaronic-based transport framework, because if carrier relaxation dominated transport, then mobility dependence on carrier density would be largely suppressed. ${ }^{[66]}$ This is therefore a very important indication regarding electron transport regime, suggesting that inter-molecular factors such as energetic disorder, rather than intra-molecular relaxation effects, could play a predominant role. For this reason we utilized energetic disorder based transport models, and in particular we analyzed the temperature dependence of the mobility in the framework of the model developed by Vissenberg and Matters (VM). ${ }^{[71]}$ In the VM model, it is assumed that transport occurs by hopping in an exponential density of states (DOS) given by $g(E)=N_{t} / k T$ exp $\left(E / k T_{0}\right)$, where $T_{0}$ is the DOS characteristic energy and is an indication of the disorder degree. The field-effect mobility is modeled as $\mu=\mu_{0}$ $\left(V_{\mathrm{g}}-V_{\mathrm{T}}\right)^{\gamma}$, where $\gamma=2\left(T_{0} / T-1\right)$, therefore depending solely on the disorder degree, and the mobility prefactor $\mu_{0}$ depends on $T_{0}$ and on $\alpha^{-1}$, the effective wavefunction overlap parameter, which is a measure of the ease of interchain hopping (see Supporting Information for more details). Results are reported in Figure 7 (and in Table S2 in the Supporting Information). As far as the effective wavefunction overlap, $\alpha^{-1}$ is largest in the case of $\mathrm{P}(\mathrm{NDI} 2 \mathrm{OD}-\mathrm{T} 2)$, then we find the co-polymers with condensed rings, P(NDI2OD-DTT) and P(NDI2OD-TT), whereas the poorest effective wavefunction overlap occurs for b)

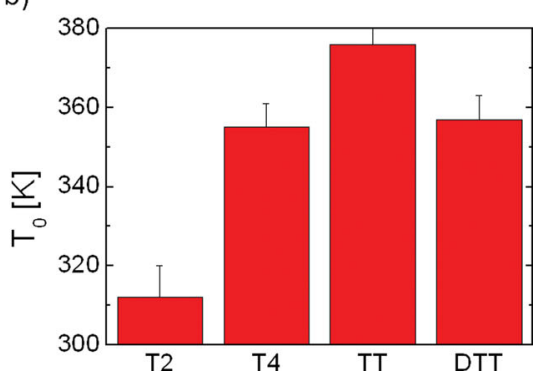

$\mathrm{P}(\mathrm{NDI}$ OD-T4). Concerning the DOS characteristic temperature $T_{0}$, it is the smallest in the case of P(NDI2OD-T2), then we find $\mathrm{P}(\mathrm{NDI} 2 \mathrm{OD}-\mathrm{DTT})$ and $\mathrm{P}(\mathrm{NDI} 2 \mathrm{OD}-\mathrm{T} 4)$, whereas it is highest in case of $\mathrm{P}(\mathrm{NDI} 2 \mathrm{OD}-\mathrm{TT})$.

$\alpha^{1}$ and $T_{0}$ can be correlated with the field-effect mobilities of the different co-polymers. The parent co-polymer P(NDI2ODT2) is characterized by the highest mobility, because it is both the least disordered, showing the lowest $T_{0}$, and because it has the largest degree of interchain coupling, showing the highest effective wavefunction overlap $\alpha^{-1}$. For this polymer the low level of energetic disorder has been previously suggested as the origin of high mobility ${ }^{[42,48]}$ In this case, also the structural characterization suggests a higher degree of order with respect to the other polymers. Among the new polymers, P(NDI2ODTT) and P(NDI2OD-DTT) show similar electrons mobility, yet lower than that of P(NDI2OD-T2). While film microstructural analysis suggests that $\mathrm{P}(\mathrm{NDI} \mathrm{OD}-\mathrm{TT})$ should be more similar to $\mathrm{P}(\mathrm{NDI} 2 \mathrm{OD}-\mathrm{T} 2)$ than $\mathrm{P}(\mathrm{NDI} 2 \mathrm{OD}-\mathrm{DTT})$, the FET mobility values for both $\mathrm{P}(\mathrm{NDI} 2 \mathrm{OD}-\mathrm{TT})$ and $\mathrm{P}(\mathrm{NDI} 2 \mathrm{OD}-\mathrm{DTT})$ are consistent with their higher degree of nanoscopic disorder and a more impeded interchain hopping with respect to P(NDI2OD-T2). Actually, P(NDI2OD-TT) appears to be the polymer among the derivatives with the highest degree of energetic disorder. This result underlines that structural analysis is important but need to be taken with care when trying to predict transport properties, as it is not trivial to derive a clear correspondence between structural data and model parameters such as $\alpha^{-1}$ and $T_{0}$. The fact that inter-molecular factors are dominating clearly appears by looking at P(NDI2OD-T4), that despite showing the lowest $\lambda_{\text {intra }}^{e}$ in the whole series, is characterized by the lowest mobility, due to a low effective wavefunction overlap. In this respect, the correlation of $\lambda_{\text {intra }}^{e}$ with the mobility for P(NDI2OD-TT) and $\mathrm{P}(\mathrm{NDI} 2 \mathrm{OD}-\mathrm{DTT})$ has to be regarded as incidental. We can therefore conclude that, in the series of devices fabricated with the newly synthesized NDI co-polymers, polaronic effects are not evident and are masked by inter-molecular effects which define the DOS and the coupling integrals. Particular attention should be paid when considering the case of the parent copolymer P(NDI2OD-T2), which represents one of the extremes of the series. Its DOS characteristic temperature is very close to room temperature $(312 \mathrm{~K} \pm 8 \mathrm{~K})$, which means that the VM model reaches the limit of applicability. Therefore in the case of such low energetic disorder, a tangible effect of carrier relaxation on transport cannot be excluded. ${ }^{[42]}$ 


\section{Conclusions}

In this work, we synthesized semiconductors consisting of an NDI unit co-polymerized with various thiophene-based donor groups and theoretically and experimentally investigated with a systematic approach their chemical-physical and charge transport properties versus those of the well-known P(NDI2OD-T2). In this series, while having the same solubilizing 2-octyldecyl side chains only on the NDI unit, strictly only the donor group was modifi ed by both fusing (P(NDI2OD-TT) and P(NDI2OD$\mathrm{DTT})$ ) and extending the number of the thiophene rings (P(NDI2OD-T4)). Our investigation has focused on the critical assessment of factors limiting n-type transport in field-effect devices based on these co-polymers, which are found to be prevalently good electron transporters. Various techniques were adopted to carefully consider both intra-molecular (frontier energy orbitals, theoretical intra-molecular reorganization energies) and inter-molecular (fi lms microstructure, investigations of the density of states through variable-temperature measurements) properties. Intra-molecular polaronic effects are predicted to be dominant only in the case of a relatively limited degree of disorder, where the reorganization energy is the key factor in limiting the electron transfer process through localized states. DFT calculations provide essential information on polaronic effects, challenging simple argumentations which can be intuitively derived from monomers, and indicate the necessity to approach these studies on suitably long model oligomers ( $n \geq 4-5$ repeat units) to reliably estimate the intra-molecular reorganization energies. Our results suggest that if polaronic effects were dominant, P(NDI2OD-T2) and P(NDI2OD-T4) should show the highest electrons mobility, since they are characterized by electron reorganization energies $\approx 0.10 \mathrm{eV}$ lower than P(NDI2OD-TT) and P(NDI2OD-DTT).

However, this picture is not confi rmed by experimental mobility values extracted from fi eld-effect devices. We have shown that while the parent P(NDI2OD-T2) show the highest electrons mobility $\left(0.1 \mathrm{~cm}^{2} \mathrm{~V}^{-1} \mathrm{~s}^{-1}\right)$, the NDI derivative with the T4 unit produces the poorest electrical performances $\left(\approx 0.01 \mathrm{~cm}^{2} \mathrm{~V}^{-1} \mathrm{~s}^{-1}\right)$ in the series. Instead P(NDI2OD-TT) and $\mathrm{P}$ (NDI2OD-DTT) based OFETs show good and intermediate electrons mobility (up to $0.04 \mathrm{~cm}^{2} \mathrm{~V}^{-1} \mathrm{~s}^{-1}$ ). In addition we have measured a sizeable dependence of mobilities on the charge density, which can be hardly justifi ed in a polaronic-dominated transport framework. Experimental fi ndings can be rationalized once inter-molecular effects are taken into account. In fact, if an effi cient coupling integral is not equivalently achieved in the solid-state for all the polymers, this can largely mask the different charge relaxation effects. Moreover, disorder can strongly modify the DOS, producing broader distributions and limiting charge transport at comparable carrier densities. We have directly addressed these aspects by analyzing variable-temperature electrical data on the basis of the well known Vissenberg and Matters model for hopping transport in an exponential DOS. This allowed us to rationalize our evidences in terms of the degree of energetic disorder and the effective wavefunction overlap, which have been demonstrated to be evidently predominant over intra-molecular polaronic effects. In this framework, P(NDI2OD-T2) shows the highest mobility because of a higher degree of structural order and facile interchain hopping. P(NDI2OD-T4) instead shows the lowest mobility mainly because of a markedly reduced effective wavefunction overlap which implies a more difficult hopping process.

Thus, in conclusion, this systematic study on an ad-hoc synthesized series of NDI based co-polymers helps to move towards the rationalization of structure-property relationships in a relevant class of semiconducting co-polymers, assisting the design of high performance electron transporting materials. The extent of the conjugation, molecular planarity and reorganization energies can provide useful design rules at the single molecule level. However modifications of a functional unit along the backbone has clearly strong solid-state implications and makes it mandatory to adopt a wide investigation approach, from the study of molecular level physical-chemical properties to solid-state effects affecting the electronic couplings and shaping the density of states, in order to correctly assess the most critical factors determining efficient charge transport in polymer field-effect devices.

\section{Experimental Section}

\subsection{Synthesis}

Preparation of Co-Polymer P(NDI2OD-TT): Under argon, a mixture of NDI2OD-Br2 (145.1 mg, $0.15 \mathrm{mmol}$ ), 2,5-bis(trimethylstannyl)thieno[3,2-b]thiophene (68.6 $\mathrm{mg}, 0.15 \mathrm{mmol})$, and $\mathrm{Pd}\left(\mathrm{PPh}_{3}\right)_{2} \mathrm{Cl}_{2}$ $(5.0 \mathrm{mg}, 0.007 \mathrm{mmol})$ in anhydrous toluene $(8 \mathrm{~mL})$ was stirred at $90{ }^{\circ} \mathrm{C}$ for 3 days. Bromobenzene $(0.3 \mathrm{~mL})$ was then added and the reaction mixture was maintained at $90{ }^{\circ} \mathrm{C}$ for an additional $12 \mathrm{~h}$. Upon cooling to room temperature, a solution of potassium fluoride $(1 \mathrm{~g})$ in water $(2 \mathrm{~mL})$ was added. This mixture was stirred and shaken at room temperature for 1 hour before it was diluted with chloroform $(150 \mathrm{~mL})$. The resulting mixture was washed with water $(60 \mathrm{~mL} \times 3)$, dried over anhydrous $\mathrm{Na}_{2} \mathrm{SO}_{4}$, and concentrated on a rotary evaporator. The residue was taken with chloroform $(30 \mathrm{~mL})$ and precipitated in methanol $(100 \mathrm{~mL})$. This procedure was repeated using chloroform and acetone, leading to a deep blue solid as the crude product. The obtained blue solid was further purified by Soxhlet extraction with acetone for $24 \mathrm{~h}$. The isolated solid residue was redissolved in chloroform $(50 \mathrm{~mL})$ and the resulting mixture was heated to boil. Upon cooling to room temperature, this chloroform solution was filtered through a syringe filter $(5 \mu \mathrm{m})$, and the filtrate was precipitated in methanol $(50 \mathrm{~mL})$. The precipitates were collected by filtration, washed with methanol, and dried in vacuum, leading to a deep blue solid as the product $(134 \mathrm{mg}$, yield $94.4 \%$ ).

${ }^{1} \mathrm{H}$ NMR $\left(\mathrm{CDCl}_{2} \mathrm{CDCl}_{2}, 500 \mathrm{MHz}\right): 8: 8.62-9.00(\mathrm{~m}, \mathrm{br}, 2 \mathrm{H}), 7.55-7.80$ $(\mathrm{m}, \mathrm{br}, 2 \mathrm{H}), 4.00-4.35(\mathrm{~m}, \mathrm{br}, 4 \mathrm{H}), 2.02(\mathrm{br}, 2 \mathrm{H}), 1.10-1.50(\mathrm{br}, 64 \mathrm{H})$ 0.80-0.95 (br, 12H). GPC: $M_{\mathrm{n}}=102.5 \mathrm{~K} \mathrm{Da}, M_{\mathrm{w}}=297.6 \mathrm{~K} \mathrm{Da}, \mathrm{PDI}=2.9$. Elemental Analysis (calc. C, 74.80; H, 9.00; N, 2.91): found C, 74.82; H, 8.89; N, 2.93.

Preparation of Co-Polymer P(NDI2OD-DTT): Under argon, a mixture of NDI2OD-Br2 (129.8 mg, $0.13 \mathrm{mmol}$ ), 5,5'-bis(trimethylstannyl)dithienothiophene $(68.8 \mathrm{mg}, 0.13 \mathrm{mmol})$, and $\mathrm{Pd}\left(\mathrm{PPh}_{3}\right)_{4}(7.6 \mathrm{mg}$, $0.0066 \mathrm{mmol})$ in anhydrous toluene $\left(12 \mathrm{~mL}\right.$ ) was stirred at $90{ }^{\circ} \mathrm{C}$ for $40 \mathrm{~h}$. Bromobenzene $(0.3 \mathrm{~mL})$ was then added and the reaction mixture was maintained at $90{ }^{\circ} \mathrm{C}$ for an additional $24 \mathrm{~h}$. Upon cooling to room temperature, a solution of potassium fluoride $(1 \mathrm{~g})$ in water $(2 \mathrm{~mL})$ was added. This mixture was stirred and shaken at room temperature for $1 \mathrm{~h}$ before it was diluted with chloroform $(150 \mathrm{~mL})$. The resulting mixture was washed with water $(80 \mathrm{~mL} \times 3)$, dried over anhydrous $\mathrm{Na}_{2} \mathrm{SO}_{4}$, and concentrated on a rotary evaporator. The residue was taken 
with chloroform $(50 \mathrm{~mL})$ and precipitated in methanol $(100 \mathrm{~mL})$. This procedure was repeated using chloroform and acetone, leading to a deep blue solid as the crude product. The obtained blue solid was further purified by Soxhlet extraction with acetone for $24 \mathrm{~h}$. The isolated solid residue was redissolved in chloroform $(80 \mathrm{~mL})$ and the resulting mixture was heated to boil. Upon cooling to room temperature, this chloroform solution was filtered through a syringe fi lter $(5 \mu \mathrm{m})$, and the filtrate was precipitated in methanol $(100 \mathrm{~mL})$. The precipitates were collected by filtration, washed with methanol, and dried in vacuum, leading to a deep blue solid as the product (91.0 $\mathrm{mg}$, yield $67.7 \%$ ).

${ }^{1} \mathrm{H}$ NMR $\left(\mathrm{CDCl}_{2} \mathrm{CDCl}_{2}, 500 \mathrm{MHz}\right): \delta: 8.60-9.00(\mathrm{~m}, \mathrm{br}, 2 \mathrm{H}), 7.50-7.90$ (m, br, 2H), 4.05-4.40 (m, br, 4H), $2.00(\mathrm{br}, 2 \mathrm{H}), 1.10-1.50(\mathrm{br}, 64 \mathrm{H})$ 0.80-0.95 (br, 12H). GPC: $M_{\mathrm{n}}=16.9 \mathrm{~K} \mathrm{Da}, M_{\mathrm{w}}=112.2 \mathrm{~K} \mathrm{Da}, \mathrm{PDI}=6.6$. Elemental Analysis (calc. C, 73.04; H, 8.50; N, 2.75): found C, 73.15; H, 8.49; N, 2.72.

Preparation of Compound T-NDI2OD-T: Under argon, a mixture of NDI2OD- $\mathrm{Br}_{2}$ (280.0 mg, $\left.0.28 \mathrm{mmol}\right)$, 2-trimethylstannylthiophene (400.0 mg, $1.62 \mathrm{mmol}), \quad \mathrm{Pd}\left(\mathrm{PPh}_{3}\right)_{2} \mathrm{Cl}_{2}(28.0 \mathrm{mg}, 0.04 \mathrm{mmol})$ in anhydrous toluene $(20 \mathrm{~mL})$ was stirred at $90^{\circ} \mathrm{C}$ for $22 \mathrm{~h}$. Upon cooling to room temperature, the reaction mixture was diluted with chloroform $(100 \mathrm{~mL})$, and the resulting mixture was washed with water $(80 \mathrm{~mL} \times$ 2), dried over anhydrous sodium sulfate $\left(\mathrm{Na}_{2} \mathrm{SO}_{4}\right)$, and concentrated on rotary evaporator. The residue was subject to column chromatography on silica gel with a mixture of chloroform:hexane $(3: 2, \mathrm{v} / \mathrm{v})$ as eluent, leading to an orange solid as the product $(240.0 \mathrm{mg}, 0.24 \mathrm{mmol}, 85.2 \%)$. ${ }^{1} \mathrm{H} \mathrm{NMR}\left(\mathrm{CDCl}_{3} 500 \mathrm{MHz}\right): \delta: 8.77(\mathrm{~s}, 2 \mathrm{H}), 7.57(\mathrm{~d}, \mathrm{~J}=5.0 \mathrm{~Hz}, 2 \mathrm{H}), 7.31$ $(\mathrm{d}, \mathrm{J}=3.5 \mathrm{~Hz}, 2 \mathrm{H}), 7.21(\mathrm{~m}, 2 \mathrm{H}), 4.07(\mathrm{~d}, \mathrm{~J}=7.5 \mathrm{~Hz}, 4 \mathrm{H}), 1.95(\mathrm{~m}, 2 \mathrm{H})$, 1.18-40 (m, br, 64H) $0.84-0.88(\mathrm{~m}, 12 \mathrm{H})$; Elemental Analysis (calc. C, 75.10; H, 9.15; N, 2.83): found C, 75.14; H, 9.10; N, 2.89 .

Preparation of Compound BrT-NDI2OD-TBr: Under argon, a mixture of T-NDI2OD-T (200.0 mg, $0.20 \mathrm{mmol}$ ) and NBS (125.0 mg, $0.70 \mathrm{mmol})$ in DMF $(20 \mathrm{~mL})$ was stirred at $80^{\circ} \mathrm{C}$ for $25 \mathrm{~h}$. Upon cooling to room temperature, reaction mixture was poured into water $(100 \mathrm{~mL})$, and the resulting mixture was extracted with chloroform $(100 \mathrm{~mL})$. Organic layer was separated, washed with water $(100 \mathrm{~mL} \times 2)$, dried over anhydrous $\mathrm{Na}_{2} \mathrm{SO}_{4}$, and concentrated on rotary evaporator. The residue was subject to column chromatography on silica gel with a mixture of chloroform:hexane $(2: 3, \mathrm{v} / \mathrm{v}$, slowly up to $1: 1)$ as eluent, leading to a red solid as the product $(145.0 \mathrm{mg}, 0.13 \mathrm{mmol}, 62.5 \%) .{ }^{1} \mathrm{H} \mathrm{NR}\left(\mathrm{CDCl}_{3}\right.$, $500 \mathrm{MHz}): \delta: 8.73(\mathrm{~s}, 2 \mathrm{H}), 7.15(\mathrm{~d}, \mathrm{~J}=4.0 \mathrm{~Hz}, 2 \mathrm{H}), 7.09(\mathrm{~d}, \mathrm{~J}=4.0,2 \mathrm{H})$, $4.08(\mathrm{~d}, \mathrm{~J}=7.5 \mathrm{~Hz}, 4 \mathrm{H}), 1.93-1.98(\mathrm{~m}, 2 \mathrm{H}), 1.20-1.40(\mathrm{br}, \mathrm{m}, 64 \mathrm{H})$, 0.83-0.89 (m, 12H). Elemental Analysis (calc. C, 64.79; H, 7.72; N, 2.44): found $\mathrm{C}, 64.50 ; \mathrm{H}, 7.74 ; \mathrm{N}, 2.49$.

Preparation of Co-Polymer P(NDI2OD-T4): Under argon, a mixture of BrT-NDI2OD-BrT (92.1 mg, $0.08 \mathrm{mmol}$ ), 5,5'-bis(trimethylstannyl)2,2'-bithiophene $(39.4 \mathrm{mg}, 0.08 \mathrm{mmol})$, and $\mathrm{Pd}\left(\mathrm{PPh}_{3}\right)_{2} \mathrm{Cl}_{2}(2.8 \mathrm{mg}$, $0.004 \mathrm{mmol})$ in anhydrous toluene $(5 \mathrm{~mL})$ was stirred at $90{ }^{\circ} \mathrm{C}$ for 4 days. Bromobenzene $(0.3 \mathrm{~mL})$ was then added and the resulting mixture was stirred for an additional 12 hours. After cooling to room temperature, a solution of potassium fluoride $(1 \mathrm{~g})$ in water $(2 \mathrm{~mL})$ was added. This mixture was stirred and shaken at room temperature for $1 \mathrm{~h}$, before it was diluted with chloroform $(150 \mathrm{~mL})$. The resulting mixture was washed with water $(100 \mathrm{~mL} \times 3)$, dried over anhydrous $\mathrm{Na}_{2} \mathrm{SO}_{4}$, and concentrated on rotary evaporator. The residue was taken with chloroform $(30 \mathrm{~mL})$ and precipitated in methanol $(50 \mathrm{~mL})$. This procedure was repeated using chloroform and acetone, leading to a dark blue solid as crude product. This crude product was purified by Soxhlet extraction with acetone for 48 hours. The isolated solid was dissolved in chloroform $(50 \mathrm{~mL})$ and then heated to boil. After cooling to room temperature, the chloroform solution was passed through a syringe filter $(5 \mu \mathrm{m})$, and the filtrate was precipitated in methanol $(50 \mathrm{~mL})$. The precipitates were collected by filtration, washed with methanol, and dried in vacuum, leading to a dark blue solid $(87.0 \mathrm{mg}$, 94.1\%). ${ }^{7} \mathrm{H} N R\left(\mathrm{CDCl}_{2} \mathrm{CDCl}_{2}, 500 \mathrm{MHz}\right): \delta: 8.70-8.81(\mathrm{~m}, \mathrm{br}, 2 \mathrm{H}), 7.10-$ $7.40(\mathrm{~m}, \mathrm{br}, 8 \mathrm{H}), 4.10(\mathrm{br}, 4 \mathrm{H}), 1.99(\mathrm{~s}, \mathrm{br}, 2 \mathrm{H}), 1.10-1.45(\mathrm{~m}, \mathrm{br}, 64 \mathrm{H})$, $0.86(\mathrm{~m}, \mathrm{br}, 12 \mathrm{H})$. GPC: $M_{\mathrm{n}}=67.4 \mathrm{~K} \mathrm{Da}, M_{\mathrm{w}}=170.3 \mathrm{~K} \mathrm{Da}, \mathrm{PDI}=2.5$. Elemental Analysis (calc. C, 72.87; H, 8.04; N, 2.43): found C, 72.69; $\mathrm{H}, 8.06 ; \mathrm{N}, 2.47$.

\subsection{Film Characterization and Device Fabrication}

For the OFETs we adopted a top-gate, bottom-contact architecture for optimized device performance. Thoroughly cleaned $1737 \mathrm{~F}$ glass was used as the substrate. Interdigitated Au contacts were defined by a lift-off photolithographic process with a $0.7 \mathrm{~nm}$ thick $\mathrm{Cr}$ adhesion layer. The thickness of the Au contacts was $30 \mathrm{~nm}$. Substrates were cleaned in a sonic bath in isopropyl alcohol for 2-3 min before deposition of the semiconductor. After filtering through a $0.2 \mu \mathrm{m}$ polytetrafluoroethylene (PTFE) filter, solutions of the polymeric semiconductors of this work (9 $\mathrm{g} \mathrm{L}^{-1}$ in 1,2-Dichlorobenzene) were deposited by spin-coating at $1000 \mathrm{rpm}$ for $90 \mathrm{~s}$ in a nitrogen glove box. The semiconductor was then annealed $\left(14 \mathrm{~h}\right.$ at $120^{\circ} \mathrm{C}$ or $30 \mathrm{~min}$ at $\left.150^{\circ} \mathrm{C}\right)$ on a hot plate in a nitrogen atmosphere. PMMA (Sigma-Aldrich) with $M_{\mathrm{w}}=120 \mathrm{~kg} \mathrm{~mol}^{-1}$ was spun from $\mathrm{n}$-butylacetate $\left(80 \mathrm{~g} \mathrm{~L}^{-1}\right.$, filtered with a $0.45 \mu \mathrm{m}$ PTFE filter) in air at $1300 \mathrm{rpm}$ for $60 \mathrm{~s}$. Dielectric-layer thicknesses $\approx 600 \mathrm{~nm}$ were obtained. After the dielectric deposition, the devices were annealed under nitrogen, on a hot-plate, at $120^{\circ} \mathrm{C}$ for $14 \mathrm{~h}$. $30 \mathrm{~nm}$ thick gate $\mathrm{Al}$ electrodes were thermally evaporated as gate contacts. The perfluorinated polymer Cytop CTL-809M dielectric (Asahi Glass) was spun as received at $9000 \mathrm{rpm}$ for $90 \mathrm{~s}$ (15 s acceleration) in air. The electrical characteristics of the transistors were measured in a nitrogen glove-box on a Wentworth Laboratories probe station with an Agilent B1500A semiconductor device analyzer and Agilent Precision LCR Meter. The OFET electrical characteristics were measured as a function of temperature using a low-temperature cryogenic probe station (Janis Laboratory Cryogenic Systems) connected to an Agilent B1500A semiconductor device analyzer. The surface morphology of the films was obtained with an Agilent 5500 Atomic Force Microscope operated in the Acoustic Mode. The thicknesses of the polymer films were measured with a KLA Tencor Alpha-Step Surface Profiler.

\section{Supporting Information}

Supporting Information is available from Wiley Online Library or from the author.

\section{Acknowledgements}

Authors are thankful to A. Petrozza for help with the variable temperature measurements, to F. Maddalena for characterization of PMMA dielectric and to Prof. C. Castiglioni and L. Brambilla for RAIRS measurements and spectra interpretation. It is acknowledged partial financial support from Fondazione Cariplo under project Indixi, Grant n. 2011-0368. M.C. acknowledges financial support from European Union through the Marie-Curie Career Integration Grant 2011 "IPPIA", within the European Union Seventh Framework Programme (FP7/2007-2013) under grant agreement $n^{\circ}$ PCIG09-GA-2011-291844. Y.Y.N. acknowledges financial support from a grant of the Centre for Advanced Soft Electronics under the Global Frontier Research Program of MEST, Korea (code no. 2013073183) and the Dongguk University Research Fund of 2013.

[1] A. C. Arias, J. D. MacKenzie, I. McCulloch, J. Rivnay, A. Salleo, Chem. Rev. 2010, 110, 3.

[2] K. J. Baeg, M. Caironi, Y. Y. Noh, Adv. Mater. 2013, DOI: 10.1002/ adma.201205361

[3] M. Caironi, E. Gili, H. Sirringhaus, in Organic Electronics II: More Materials and Applications, (Ed: H. Klauk), Wiley VCH, 2012, 281. 
[4] A. J. Kronemeijer, E. Gili, M. Shahid, J. Rivnay, A. Salleo, M. Heeney, H. Sirringhaus, Adv. Mater. 2012, 24, 1558.

[5] R. R. Søndergaard, M. Hösel, F. C. Krebs, J. Polym, Sci., Part B: Polym. Phys. 2013, 51, 16.

[6] H. Sirringhaus, M. Bird, N. Zhao, Adv. Mater. 2010, 22, 3893.

[7] H. Klauk, Chem. Soc. Rev. 2010, 39, 2643.

[8] L. Basiricò, P. Cosseddu, A. Scidà, B. Fraboni, G. G. Malliaras, A. Bonfiglio, Org. Electron. 2012, 13, 244.

[9] G. Gelinck, P. Heremans, K. Nomoto, T. D. Anthopoulos, Adv. Mater. 2010, 22, 3778.

[10] J. Rivnay, S. C. B. Mannsfeld, C. E. Miller, A. Salleo, M. F. Toney, Chem. Rev. 2012, 112, 5488.

[11] M. Muccini, Nat. Mater. 2006, 5, 605.

[12] H. N. Tsao, D. Cho, J. W. Andreasen, A. Rouhanipour, D. W. Breiby, W. Pisula, K. Müllen, Adv. Mater. 2009, 21, 209.

[13] H. N. Tsao, D. M. Cho, I. Park, M. R. Hansen, A. Mavrinskiy, D. Y. Yoon, R. Graf, W. Pisula, H. W. Spiess, K. Müllen, J. Am. Chem. Soc. 2011, 133, 2605.

[14] Y. Li, S. P. Singh, P. Sonar, Adv. Mater. 2010, 22, 4862.

[15] J. S. Ha, K. H. Kim, D. H. Choi, J. Am. Chem. Soc. 2011, 133, 10364.

[16] Y. Li, P. Sonar, S. P. Singh, W. Zeng, M. S. Soh, J. Mater. Chem. 2011, 21, 10829

[17] H. Bronstein, Z. Chen, R. S. Ashraf, W. Zhang, J. Du, J. R. Durrant, P. Shakya Tuladhar, K. Song, S. E. Watkins, Y. Geerts, M. M. Wienk, R. A. J. Janssen, T. Anthopoulos, H. Sirringhaus, M. Heeney, I. McCulloch, J. Am. Chem. Soc. 2011, 133, 3272.

[18] I. Osaka, T. Abe, S. Shinamura, K. Takimiya, J. Am. Chem. Soc. 2011, $133,6852$.

[19] T. Lei, Y. Cao, Y. Fan, C.-J. Liu, S.-C. Yuan, J. Pei, J. Am. Chem. Soc. 2011, 133, 6099.

[20] J. Mei, D. H. Kim, A. L. Ayzner, M. F. Toney, Z. Bao, J. Am. Chem. Soc. 2011, 133, 20130.

[21] H. Yan, Z. Chen, Y. Zheng, C. Newman, J. R. Quinn, F. Dotz, M. Kastler, A. Facchetti, Nature 2009, 457, 679.

[22] J. Lee, A. R. Han, H. Yu, T. J. Shin, C. Yang, J. H. Oh, J. Am. Chem. Soc. 2013, 135, 9540.

[23] J. H. Park, E. H. Jung, J. W. Jung, W. H. Jo, Adv. Mater. 2013, 25, 2583.

[24] J. Li, Y. Zhao, H. S. Tan, Y. Guo, C.-A. Di, G. Yu, Y. Liu, M. Lin, S. H. Lim, Y. Zhou, H. Su, B. S. Ong, Sci. Rep. 2012, 2.

[25] B. A. Jones, A. Facchetti, M. R. Wasielewski, T. J. Marks, J. Am. Chem. Soc. 2007, 129, 15259.

[26] R. P. Ortiz, H. Herrera, R. Blanco, H. Huang, A. Facchetti, T. J. Marks, Y. Zheng, J. L. Segura, J. Am. Chem. Soc. 2010, 132, 8440.

[27] J. Cornil, J. L. Brédas, J. Zaumseil, H. Sirringhaus, Adv. Mater. 2007, 19, 1791.

[28] T. D. Anthopoulos, G. C. Anyfantis, G. C. Papavassiliou, D. M. de Leeuw, Appl. Phys. Lett. 2007, 90, 122105.

[29] M. L. Tang, Z. Bao, Chem. Mater. 2010, 23, 446.

[30] B. J. Jung, N. J. Tremblay, M.-L. Yeh, H. E. Katz, Chem. Mater. 2010, 23, 568.

[31] F. Wurthner, M. Stolte, Chem. Commun. 2011, 47, 5109.

[32] X. Zhan, A. Facchetti, S. Barlow, T. J. Marks, M. A. Ratner, M. R. Wasielewski, S. R. Marder, Adv. Mater. 2011, 23, 268.

[33] A. Babel, S. A. Jenekhe, J. Am. Chem. Soc. 2003, 125, 13656.

[34] X. Zhan, Z. a. Tan, B. Domercq, Z. An, X. Zhang, S. Barlow, Y. Li, D. Zhu, B. Kippelen, S. R. Marder, J. Am. Chem. Soc. 2007, 129, 7246.

[35] F. Würthner, V. Stepanenko, Z. Chen, C. R. Saha-Möller, N. Kocher, D. Stalke, J. Org. Chem. 2004, 69, 7933.

[36] C. Thalacker, C. Röger, F. Würthner, J. Org. Chem. 2006, 71, 8098.

[37] X. Guo, M. D. Watson, Org. Lett. 2008, 10, 5333.

[38] F. S. Kim, X. Guo, M. D. Watson, S. A. Jenekhe, Adv. Mater. 2010, $22,478$.
[39] R. Kim, P. S. K. Amegadze, I. Kang, H.-J. Yun, Y.-Y. Noh, S.-K. Kwon, Y.-H. Kim, Adv. Funct. Mater. 2013, in press, DOI: 10.1002/adfm.201301197

[40] Z. Chen, Y. Zheng, H. Yan, A. Facchetti, J. Am. Chem. Soc. 2009, $131,8$.

[41] J. Li, J. Du, J. Xu, H. L. W. Chan, F. Yan, Appl. Phys. Lett. 2012, 100, 033301.

[42] M. Caironi, M. Bird, D. Fazzi, Z. Chen, R. Di Pietro, C. Newman, A. Facchetti, H. Sirringhaus, Adv. Funct. Mater. 2011, 21, 3371.

[43] J. Rivnay, M. F. Toney, Y. Zheng, I. V. Kauvar, Z. Chen, V. Wagner, A. Facchetti, A. Salleo, Adv Mater 2010, 22, 4359.

[44] R. Steyrleuthner, M. Schubert, F. Jaiser, J. C. Blakesley, Z. Chen, A. Facchetti, D. Neher, Adv. Mater. 2010, 22, 2799.

[45] S. Fabiano, C. Musumeci, Z. Chen, A. Scandurra, H. Wang, Y.-L. Loo, A. Facchetti, B. Pignataro, Adv. Mater. 2012, 24, 951.

[46] C. J. Takacs, N. D. Treat, S. Krämer, Z. Chen, A. Facchetti, M. L. Chabinyc, A. J. Heeger, Nano Lett. 2013, 13, 2522.

[47] C. B. Nielsen, M. Turbiez, I. McCulloch, Adv. Mater. 2012, 25, 1859.

[48] D. Fazzi, M. Caironi, C. Castiglioni, J. Am. Chem. Soc. 2011, 133, 47.

[49] E. Giussani, D. Fazzi, L. Brambilla, M. Caironi, C. Castiglioni, Macromolecules 2013, 46, 2658.

[50] G.-J. A. H. Wetzelaer, M. Kuik, Y. Olivier, V. Lemaur, J. Cornil, S. Fabiano, M. A. Loi, P. W. M. Blom, Phys. Rev. B 2012, 86, 165203.

[51] X. Guo, F. S. Kim, M. J. Seger, S. A. Jenekhe, M. D. Watson, Chem. Mater. 2012, 24, 1434.

[52] M. M. Durban, P. D. Kazarinoff, C. K. Luscombe, Macromolecules 2010, 43, 6348.

[53] D. L. Cheung, A. Troisi, Phys. Chem. Chem. Phys. 2008, 10, 5941.

[54] B. Baumeier, J. Kirkpatrick, D. Andrienko, Phys. Chem. Chem. Phys. 2010, 12, 11103

[55] R. P. Ortiz, H. Herrera, C. Seoane, J. L. Segura, A. Facchetti, T. J. Marks, Chem.-Eur. J. 2012, 18, 532.

[56] R. P. Ortiz, A. Facchetti, T. J. Marks, J. Casado, M. Z. Zgierski, M. Kozaki, V. Hernández, J. T. L. Navarrete, Adv. Funct. Mater. 2009, 19, 386.

[57] J. Casado, V. Hernández, O.-K. Kim, J.-M. Lehn, J. T. López Navarrete, S. Delgado Ledesma, R. Ponce Ortiz, M. C. Ruiz Delgado, Y. Vida, E. Pérez-Inestrosa, Chem.-Eur. J. 2004, 10, 3805.

[58] A. Troisi, Chem. Soc. Rev. 2011, 40, 2347.

[59] S. Stafström, Chem. Soc. Rev. 2010, 39, 2484.

[60] D. L. Cheung, D. P. McMahon, A. Troisi, J. Am. Chem. Soc. 2009, 131, 11179.

[61] C. Poelking, E. Cho, A. Malafeev, V. Ivanov, K. Kremer, C. Risko, J.-L. Brédas, D. Andrienko, J. Phys. Chem. C 2013, 117, 1633.

[62] M. Caironi, C. Newman, J. R. Moore, D. Natali, H. Yan, A. Facchetti, H. Sirringhaus, Appl. Phys. Lett. 2010, 96, 183303.

[63] Z. Chen, Y. Zheng, H. Yan, A. Facchetti, J. Am. Chem. Soc. 2008, $131,8$.

[64] R. Steyrleuthner, M. Schubert, I. Howard, B. Klaumünzer, K. Schilling, Z. Chen, P. Saalfrank, F. Laquai, A. Facchetti, D. Neher, J. Am. Chem. Soc. 2012, 134, 18303.

[65] I. H. Nayyar, E. R. Batista, S. Tretiak, A. Saxena, D. L. Smith, R. L. Martin, J. Phys. Chem. Lett. 2011, 2, 566.

[66] I. I. Fishchuk, A. Kadashchuk, V. N. Poroshin, H. Bässler, Philosoph. Mag. 2010, 90, 1229.

[67] J. Veres, S. Ogier, G. Lloyd, D. de Leeuw, Chem. Mater. 2004, 16, 4543.

[68] H. Yan, T. Schuettfort, A. J. Kronemeijer, C. R. McNeill, H. W. Ade, Appl. Phys. Lett. 2012, 101, 093308.

[69] K.-J. Baeg, D. Khim, S.-W. Jung, M. Kang, I.-K. You, D.-Y. Kim, A. Facchetti, Y.-Y. Noh, Adv. Mater. 2012, 24, 5433.

[70] D. Natali, L. Fumagalli, M. Sampietro, J. Appl. Phys. 2007, 101, 014501.

[71] M. Vissenberg, M. Matters, Phys. Rev. B 1998, 57, 12964. 